\title{
HIGH-FREQUENCY AND VERY-HIGH-FREQUENCY (HF \& VHF) ABOVE-GROUND ELECTROMAGNETIC IMPEDANCE MEASUREMENTS
}

\author{
William Frangos ${ }^{1}$, Alex Becker ${ }^{2}$, and K. H.Lee ${ }^{2}$ \\ ${ }^{1}$ Dept. of Geology and Environmental Science, James Madison \\ University, Harrisonburg, VA22801 \\ ${ }^{2}$ Lawrence Berkeley National Laboratory, Berkeley, CA 94720
}

\begin{abstract}
We have field-tested an apparatus for measuring the electromagnetic impedance above the ground at a plurality of frequencies in the $0.3-30 \mathrm{MHz}$ range. This window in the frequency spectrum, which lies between frequencies used for GPR and those used for conventional loop-loop EM soundings, has not been used because of difficulties in fielding equipment for making absolute and accurate measurements. Model and physical parameter studies however confirm that data in this frequency band can be used to construct high-resolution maps of electrical conductivity and permittivity of near-surface material. Our equipment was assembled using commercial electric and magnetic antennas. The magnetic loop source is excited by a conventional signal generator - power amplifier assembly. Signal detection is accomplished using RF lock-in amplifiers. All system elements are appropriately isolated by optic - fiber links. We estimate a measurement accuracy of about $\pm 10 \%$ for an 8-m separation between source and detector. Field tests were done at the University of California Richmond Field Station where the near surface electrical structure is well known. The experimental data at this site are mainly a function of electrical conductivity. In this context, we have obtained good agreement with the known local variations in resistivity both with depth and with position along a 35-m traverse. Additional tests in more resistive regimes where dielectric permittivity is not negligible yield spectral data compatible with the less well known near-surface electrical properties.
\end{abstract}

\section{Introduction}

Knowledge of the electrical properties of near-surface regions can help in engineering and environmental studies, both as an indication of other physical properties and as a means of detecting inhomogeneities. Compared to traditional geophysical problems, engineering and environmental issues are often small scale and shallow, so much so that for many years little geophysical effort was expended on investigations where better information was more easily gained by excavation. Recently however, problems have been recognized in which non-invasive investigations are preferred. Among these are included situations where excavation would be hazardous, cosmetically offensive, or expensive compared to the value of the required information. 
Electromagnetic induction methods have been developed for determining subsurface electrical properties for traditional geophysical problems. Typical depths of investigation and frequencies of operation of several classes of electric and electromagnetic geophysical methods are summarized in Figure 1. Note that conduction effects dominate the electrical behavior of earth materials at frequencies below a few megahertz, while dielectric effects control it at higher frequencies.

Traditionally, DC resistivity and loop-loop induction measurements with small spacings have served adequately to determine very near-surface resistivity, while groundpenetrating radar (GPR) has been used to investigate shallow subsurface permittivity contrasts. The DC resistivity and induction methods, while not limited as to depth of investigation, do suffer from lack of resolution and ambiguity of the significance of the resistivity property. While GPR can provide resolution unparalleled in geophysical practice, the method is often severely limited in its depth of investigation and, as frequently practiced, produces qualitative images with little information concerning subsurface intrinsic physical properties. The depth limitations of GPR originate from the attenuation of high frequency electromagnetic waves in conductive media. In efforts to overcome this limitation, progressively lower frequency GPR systems have been tested. Unfortunately, these systems are cumbersome and yield lower resolution than traditional GPR, negating the usual practical advantages of the method. DC resistivity methods, as practiced, tend to be slow and expensive in application. Furthermore, the general requirement of planting electrodes in the earth, in addition to being operationally costly, can be undesirably invasive. Traditional inductive EM techniques are almost as rapid as GPR but are frequently less informative concerning geometric information, particularly in complex environments.

A high-frequency impedance (HFI) system offers the possibilities of determining both resistivity and permittivity at depths down to a few meters, providing a quantitative volume-averaged, synoptic view of subsurface properties, as well as being rapid and inexpensive in the field. As seen in Figure 1, the method bridges the spectral gap between traditional electromagnetic methods and GPR. Accordingly, the HFI method requires consideration of both resistive and dielectric (permittive) earth properties and both diffusion and propagation phenomena. Song, et al. (2002) provide an analysis of a plane-wave technique to operate in this spectral region. As a practical matter, generating plane wave at these frequencies is inconvenient because of interference with other users of the electromagnetic spectrum. The HFI system described here has been implemented in a proof-of-concept form, utilizing off-the-shelf instrumentation, and tested in controlled field conditions. The new system determines the electromagnetic impedance at frequencies between $100 \mathrm{kHz}$ and $30 \mathrm{MHz}$ from direct measurement of continuous wave electric and magnetic fields at source-to-detector separations between four and sixteen meters. 


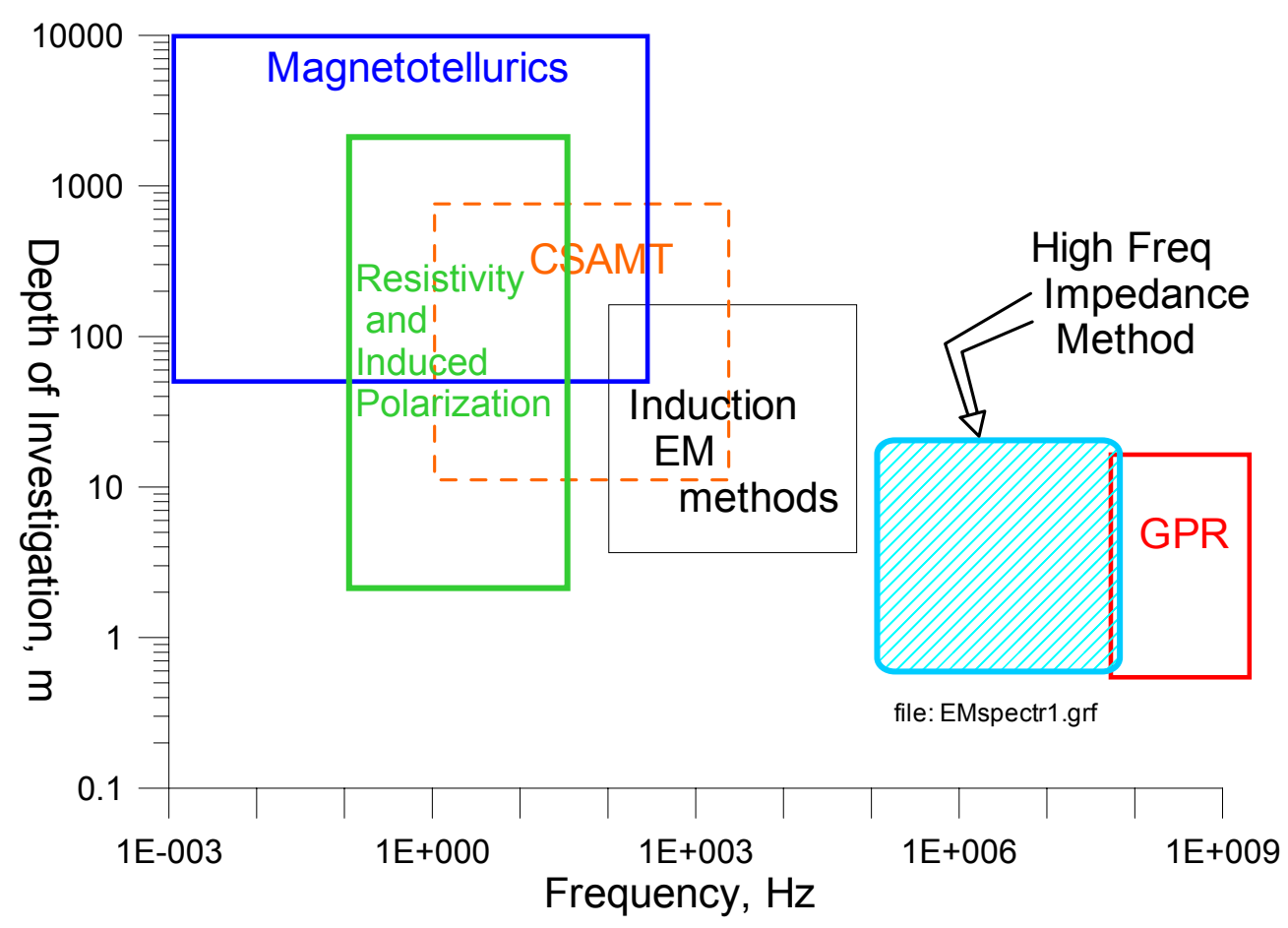

Figure 1: Ranges of applicability of electrical geophysical methods

\section{Basic Theory}

The electrical properties of soils and clays may be determined remotely by measurement of electric and magnetic fields on or above the ground surface. The electrical conductivity and dielectric permittivity determine the behavior of the fields through the complex propagation constant, $\mathrm{k}$. The measurable fields may be used to infer subsurface electrical properties.

The plane wave characteristic impedance, $Z$, of a homogeneous isotropic medium is defined in its general form as

$$
Z=\frac{E_{x}}{H_{y}}=\frac{\mu \omega}{k}=\frac{\mu \omega}{\sqrt{\varepsilon \mu \omega^{2}-i \mu \sigma \omega}}
$$

where $\quad k(\omega)=\sqrt{\varepsilon \mu \omega^{2}-i \mu \sigma \omega}$

is the spatial wave number, a complex vector with magnitude equal to the medium propagation constant,

$\varepsilon$ is the dielectric permittivity,

$\mu$ is the magnetic permeability,

$\sigma$ is the electric conductivity,

$\omega$ is the angular frequency, and

$i=\sqrt{-1}$ is the imaginary operator. 
Equation (3) can be rationalized and rewritten as,

$$
Z^{2}=\mu_{0}\left[\frac{\varepsilon}{\varepsilon^{2}+\frac{\sigma^{2}}{\omega^{2}}}+i \frac{\sigma / \omega}{\varepsilon^{2}+\frac{\sigma^{2}}{\omega^{2}}}\right]
$$

We note that the real term is controlled by the dielectric permittivity and the imaginary term by the conductivity.

The surface impedance of a two-layered earth may be calculated as shown by Wait (1970), and by Ward and Hohmann (1987), using the full expression for the wavenumber, $k$.

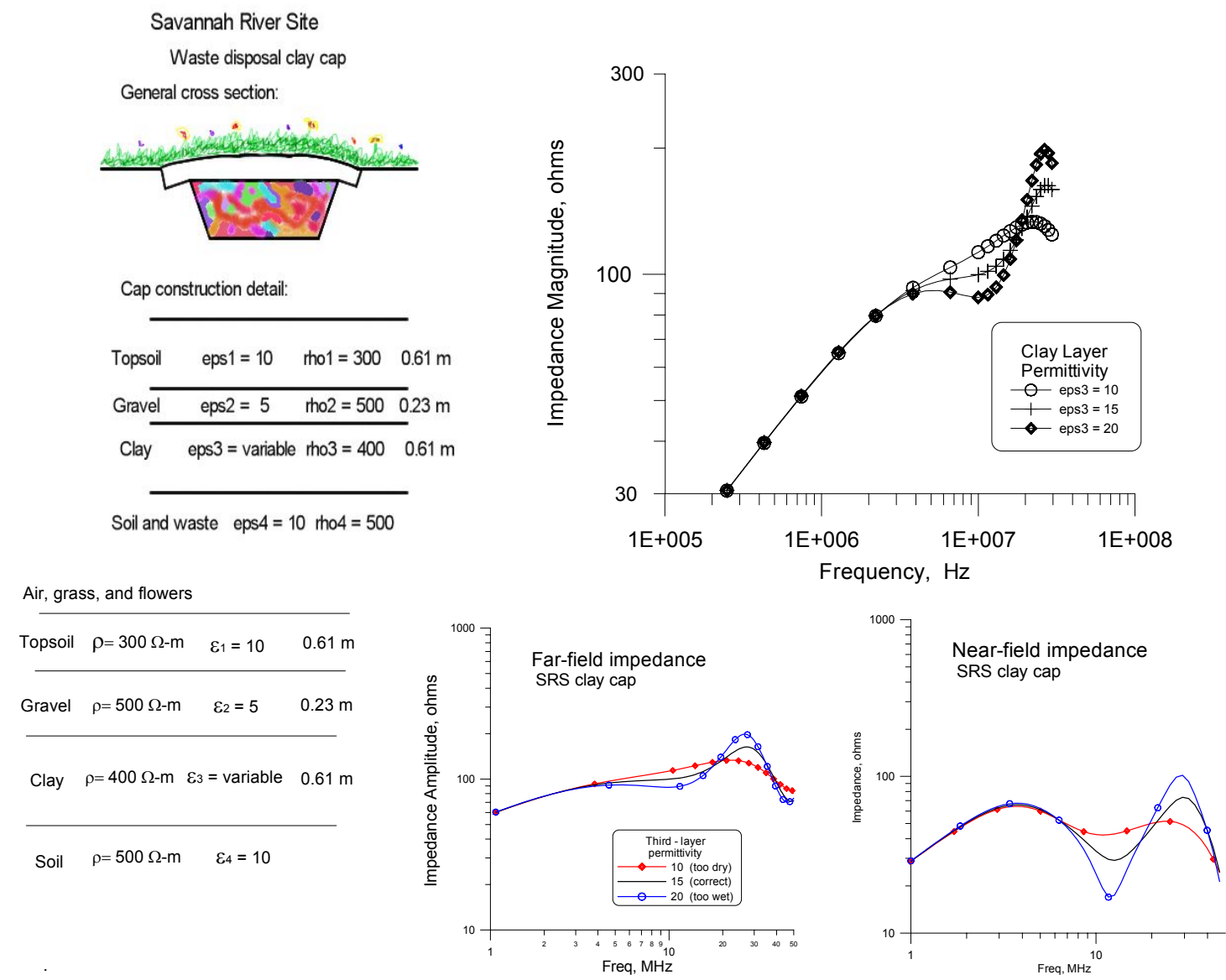

Figure 2: a) Sketch of the Savannah River Site clay cap structure, b) calculated vertical-incidence plane-wave surface impedance over hypothetical SRS clay cap at shrinkage-limit, optimum, and liquid-limit molding water contents, c) calculated near-field impedance, detector and transverse horizontal magnetic dipole source $1 \mathrm{~m}$ above the surface and $8 \mathrm{~m}$ apart

As an example, consider a case based on the protective clay caps at the Savannah 
River Site (SRS) nuclear waste disposal ponds. Figure $2 \mathrm{~b}$ shows calculated model results of the surface impedance over a four-layer earth whose third layer, the clay cap, has permittivities corresponding to deficient, optimal, and excess water content. For the vertically incident plane-wave case, significant differences of $10-20 \%$ occur between the curves corresponding to optimal and flawed third-layer water contents at frequencies between 10 and $30 \mathrm{MHz}$. This finding is highly encouraging to the concept of noninvasive monitoring of the moisture condition of clay caps at SRS through high frequency impedance measurements.

The plane-wave solutions are useful for gaining an understanding of the general field behavior, but in practice, power and signal strength considerations require the use of finite sources with relatively close separations between the transmitter and receiver. We employ the EM1D layered-earth modeling program for these cases and refer to the ratio $Z=E_{x} / H_{y}$ as the near-field impedance. Figure 2c shows the near-field impedance spectrum for the SRS clay cap model as above. Note that the differences between the spectra are even more pronounced for the same variations in third-layer permittivity than with plane-wave illumination.

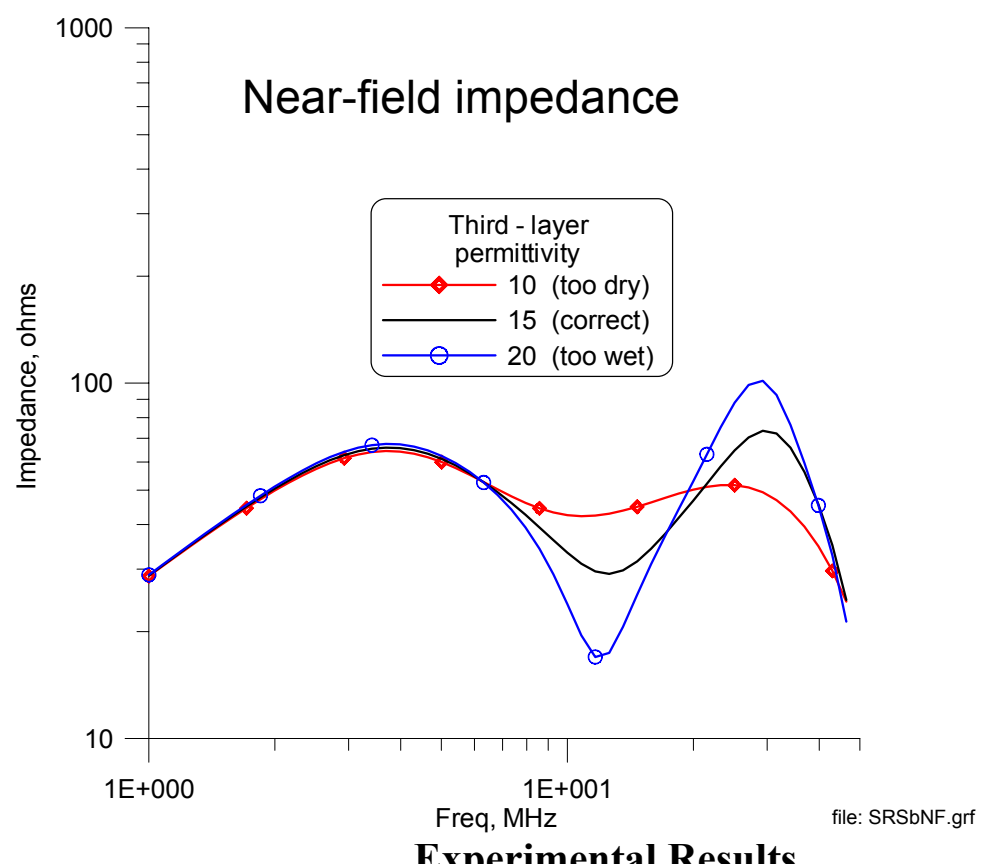

Quantitative measurement of high-frequency electromagnetic fields near the earth's surface is a difficult task. Radio engineers have directed their efforts toward minimizing the influence of the earth on system behavior. In applied geophysics, just the opposite is desired. Specifically, we wish to make measurements in such a fashion that they yield the most possible information about some designated aspect of the subsurface with the least amount of painstaking experimental procedure necessary to avoid other, interfering influences. 


\section{Measurement System}

A system based on commercially available equipment was assembled to generate and measure electromagnetic fields in the frequency range $100 \mathrm{kHz}$ through $30 \mathrm{MHz}$. A salient feature is the deployment of the antennas above the surface of the earth to minimize ground-loading effects on the source and sensor. Figure 3 is a block diagram of the measurement system.

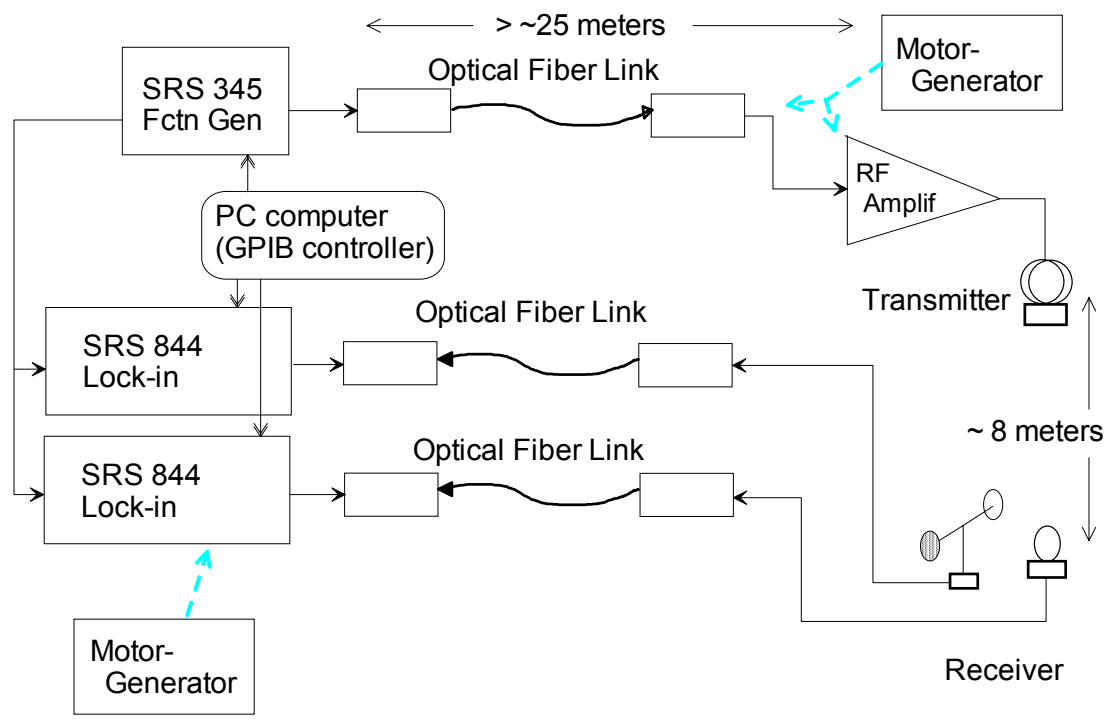

Figure 3: Block diagram of measurement system

Data were taken at the University of California's Richmond Field Station in an area of distributary Holocene sediments. At the time of the trials, the resistivity of the near-surface soils and sediments was highly variable, as shown in Figure 4, with the general characteristic that the upper region was drier and thus more resistive and less permittive than the moister lower strata. A shallow Schlumberger sounding at the site of the measurements below revealed approximately $25 \mathrm{~cm}$ of $32 \Omega-\mathrm{m}$ material overlying a basement of $10 \Omega-\mathrm{m}$. 


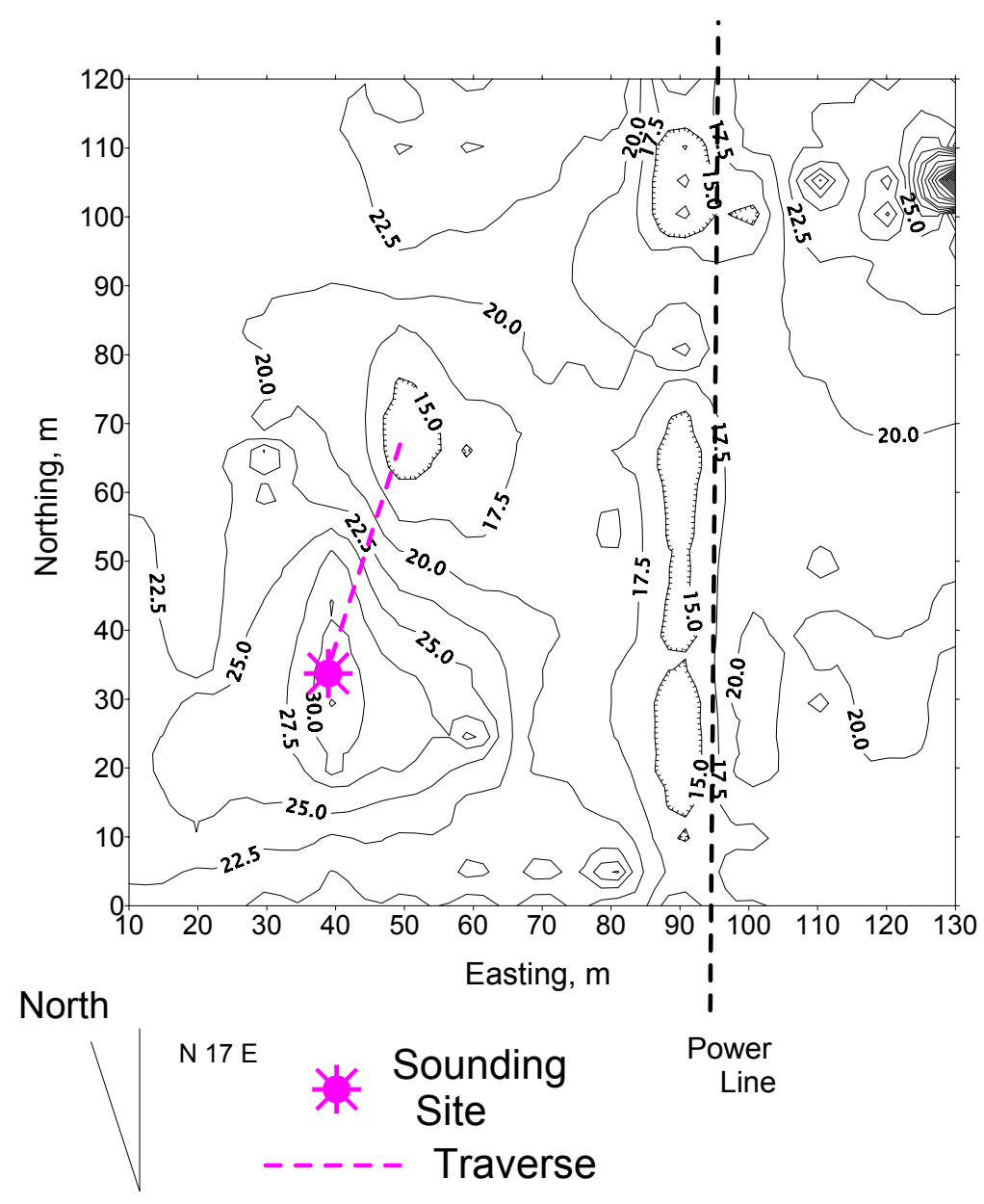

Figure 4: EM-31 Apparent resistivity of Richmond Field Station test area, in $\Omega-\mathrm{m}$

\section{Observations in a conductive environment}

The electromagnetic impedance, $Z_{x y}=\left|E_{x} / H_{y}\right|$, was measured with a transverse magnetic source, $M_{y}$, situated $8 \mathrm{~m}$ from the receiver; both antennae were $1.2 \mathrm{~m}$ above the surface. The observed data are shown with the expected response of an inverted model in Figure 5. The fit between the observed data and the forward calculation of the inverted model is good through the middle frequencies, deteriorating at the extremes where signal levels were limited by the RF amplifier response and, at the low-frequency end, by interference from a nearby military transmitter. In this conductive environment and frequency range, the dielectric properties of the earth are of little consequence to the impedance and could not be interpreted meaningfully.

Similar data taken along the traverse indicated in Figure 4 show the resistive upper layer becoming thinner and more conductive to the northeast, in agreement with shallow Schlumberger soundings. Additional data are available in Frangos (2000). 


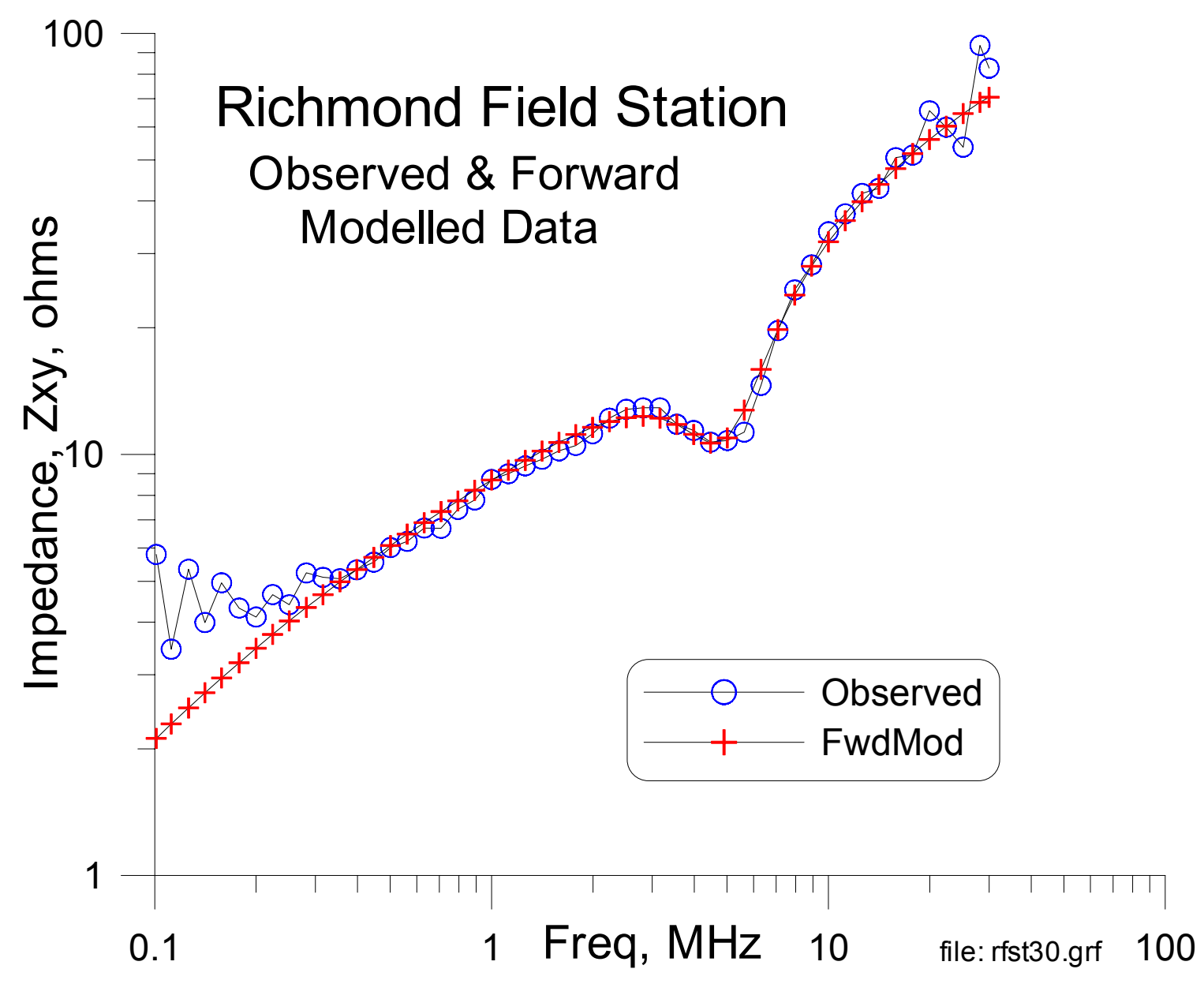

Figure 5: Observed electromagnetic impedance data from Richmond Field Station and an inverted model

\section{Observations in a resistive environment}

Determination of permittivity as an indication of water content can be expected within more resistive environments than at the Richmond Field Station. To this end we took data at Donner Pass, California, during the winter. The low-frequency resistivity of the frozen Sierra granodiorite outcrop was about 2,000 $\Omega-\mathrm{m}$; beyond this, the ground was not well characterized. The permittivity may be expected to be fairly low since the interstitial water was largely frozen and the constituent minerals have relative permittivities of 4 to 8 . The transmitter magnetic dipole was oriented both transverse to the receiver, as in the previous example, and vertically. Representative spectra for each orientation are shown in Figure 6. 

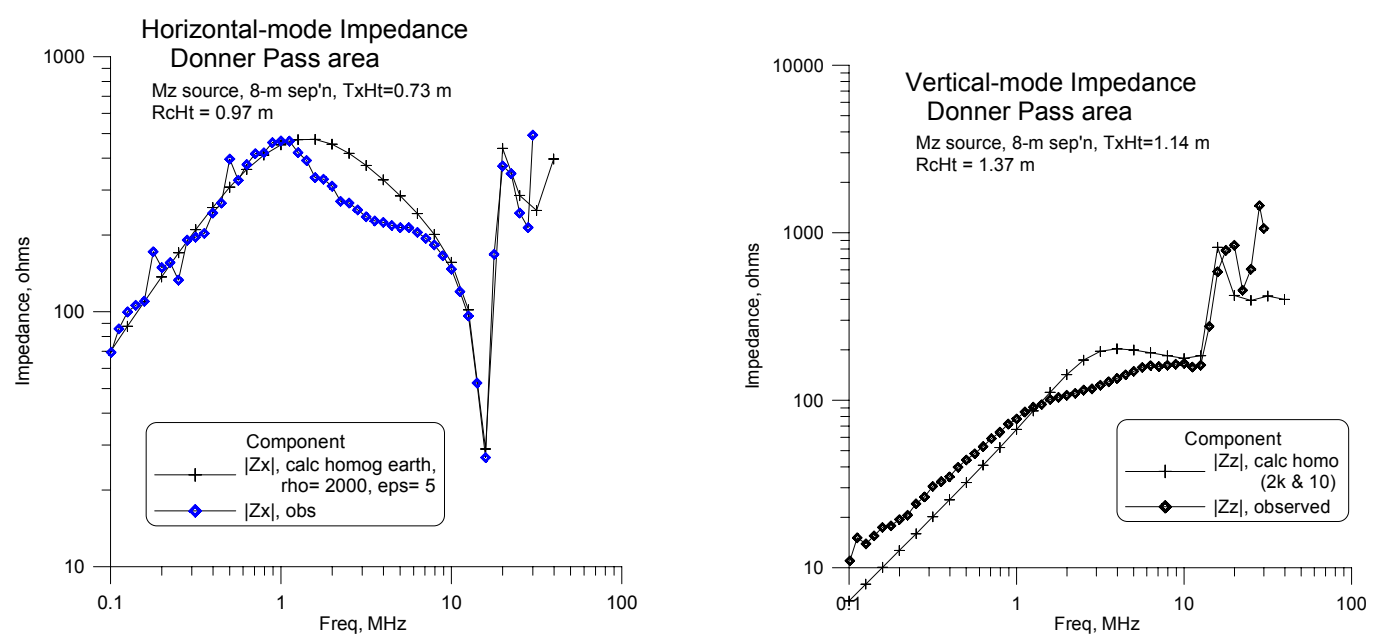

Figure 6: Electromagnetic impedance spectra from the high-resistivity Donner Pass area.

a) Transverse transmitter dipole, $Z_{x}=E_{x} / H_{y}$ b) Vertical transmitter, $Z_{z}=E_{y} / H_{z}$

Source and sensor heights above ground level as noted.

In both instances shown in Figure 6, there is a good overall agreement between the observed data and that expected for a homogeneous earth of 2,000 $\Omega-\mathrm{m}$ and low permittivity. The reproduction of the intricate behavior above $10 \mathrm{MHz}$ is particularly good, while both observed spectra display a mismatch between 2 and $8 \mathrm{MHz}$.

Considering the lack of detailed knowledge of the bedrock physical properties, the data support the viability of the system for measuring the electric and magnetic fields in a resistive environment.

\section{Conclusions}

Electromagnetic impedance measurements in the frequency range $0.1-30 \mathrm{MHz}$ can be useful in determining subsurface electrical resistivity and dielectric permittivity. We have demonstrated a proof-of-concept system for making the necessary measurements of electric and magnetic fields. Impedance data taken with the system agree well with the known local resistivity variations along a traverse in a conductive environment. Adequate agreement between observed and calculated impedances is also demonstrated in a resistive environment, where permittivity is the controlling electrical property.

\section{Acknowledgment}

This work was supported by the Assistant Secretary for Environmental Restoration and Waste Management, Office of Technology Development of the U.S. Department of Energy under contract No. DE-AC03-76SF00098.

\section{References}


Frangos, W., 2000, High frequency impedance measurements for non-invasive permittivity determination: Ph.D. dissertation, University of California, Berkeley

Song, Y., Kim, H.J., and Lee, K.H., 2002, High-frequency electromagnetic method for subsurface imaging; Geophysics, vol. 67, No. 2, pp. 501-510

Wait, J.R., 1970, Electromagnetic waves in stratified media: Pergamon Press, Oxford

Ward, S.H. and Hohmann,G.W., 1987, Electromagnetic theory for geophysical applications, in Electromagnetic methods in applied geophysics, Misac N. Nabighian, Editor; Soc. Expl. Geoph., Tulsa 